\title{
BACTERIAL TRANSLOCATION: HISTORY, MODERN APPROACHES, SCIENTIFIC AND PRACTICAL IMPORTANCE
}

\author{
Aheyev V. O., Kravchenko N. O., Bozhok L. V., Golovach O. V. \\ Institute of Agricultural Microbiology and Agricultural Production NAAS \\ 97, Shevchenko, Str., Chernihiv, 14027, Ukraine \\ E-mail: iggardsil@mail.ru
}

Given review is briefly summarizing published data on the history and current state such phenomenon as bacterial translocation. The basic patterns of saprophytic bacteria translocation, as well as theoretical and practical importance of further study of this phenomenon are described.

Keywords: bacterial translocation, normal microflora, probiotics, nonspecific resistance, treatment.

Comprehensive knowledge of all aspects and mechanisms of interaction of the living animal organism with its own microflora is one of the most fundamental problems of modern biological, medical and veterinary science an extensive.

Research history. Since the origins of microbiology and for a long time the conception of interaction depth of macroorganism with its microflora was limited to the confidence that representatives of normal microbiota in all its diversity exist exclusively on the external surfaces of the macroorganism, mainly in the epithelium of the gastrointestinal tract (GIT). The presence of any microorganisms in the blood or in tissues of parenchymal organs was interpreted only as an indication of progressive pathological condition and (or) as a result of a significant weakening of the organisms protective capacity [1].

Only in the late twentieth century some messages have appear with the new discovered facts and partial explanation of new aspects and relationships dependences of macro- and microorganisms. New scientific data allowed an unconventional look both at the problem in general and at the issue of creation and introduction of probiotic preparations.

At the end of the XIX century renowned surgeon W. Coley paid attention to the fact that cancer patients with opportunistic infections had partial "resorption" of malignant tumor. Possible causes of this phenomenon by that time remained unexplained [2]. Only in the 80 -s of XX century, almost after a century later, the number of experimental data have grown in foreign scientific literature, contradicting the traditional view of the mechanisms of probiotics action on animals and humans. Authors from different countries have observed cases when probiotic preparations act effectively that had no explanation in terms of conventional point of view. Thus, many reports have presented data on the effectiveness of bacterial preparations created based on live probiotic microorganisms representatives of the gastrointestinal microflora, used not only to treat infections of the digestive tract by intestinal colonization, but also in the treatment of pathological processes located outside the gastrointestinal tract, namely: external injuries, postoperative suppuration, pyelonephritis, respiratory diseases etc. 
Nevertheless, such effect of probiotic microorganisms was not always possible to be explained by the action of biologically active metabolites that after getting into the bloodstream can stimulate adaptive and protective reactions of macroorganism.

Attempting to explain such "non-standard" efficacy of probiotics, the hypothesis on the possibility of penetration of microbial cells through mucous membranes into the bloodstream and lymph of animals and, accordingly, into the tissues and organs including the lesions and sites of tissue damage, where the therapeutic effect by bacterial preparations (acceleration of clarification, regeneration and epithelialization processes of necrotic tissues), has arisen [3, 4].

It found its experimental evidence while discovered phenomenon of bacterial penetration through the intact wall of the gastrointestinal tract into the blood and parenchymal organs was called "bacterial translocation" and first described by American researcher R. D. Berg in 1985 [5].

At the beginning of the XXI century professor V.I. Nikitenko et al. (2004) using radioactive labeled $\mathrm{H}^{3}$-leucine two hay strains have demonstrated the ability of probiotic bacteria translocation from the gastrointestinal tract into the blood and lymph flow [6].

Modern approaches on bacterial translocation phenomenon. According to V. I. Nikitenko (1990), V. V. Smirnov et al. (1993), V. A. Kopylov and V. V. Zakharov (2002), translocation may be a natural defense mechanism and is conditioned by macroorganism functions, but not the bacterial properties. Thus, there are evidences that bacterial translocation of probiotic strains and caused by it short-term asymptomatic bacteremia may be one of the important factors that activate and maintain on required level nonspecific resistance of the macroorganism [7, 8].

Some dependences of bacterial translocation in animals were clarified and analyzed in detail by scientists of the D. K. Zabolotny Institute of Microbiology and Virology NAS Ukraine (V. V. Smirnov et al., 1993) [9].

V.I. Nikitenko first in the world has suggested using live bacteria, especially aerobic bacilli, for prevention and treatment of surgical infections, applying them orally [7].

Nowadays there are two "camps" of scientists devided by their relation to the bacterial translocation phenomenon. Some of them believe that any penetration of bacteria from the digestive tract into the bloodstream is sign of acute infection, even in case of lactic acid bacteria from dairy products [10-13]. Scientists of this group are even looking for possible ways to inhibit these processes [14]. However, the safety of probiotic strains was proved by a series of experiments on laboratory animals, intravenously introduced to these bacteria, including hay bacillus, at a dose of 170 billion microbial cells causing neither animal death nor illness. Autopsy of animals withdrawn from the experiment have shown no inflammations of internal organs or their membranes. The matter of fact, bacteria consider non-pathogenic with $\mathrm{LD}_{50}$ for mice over or equal to 2 billion living cells [6].

Other researchers, which form majority today are inclined toward an alternative point of view, which is based on the fact that bacterial translocation phenomenon is a sign of stable symbiotic relationships of animal with its microbiota $[15,16]$.

This point of view was also proved by the fact that not only selected bacterial strains with high physiological and therapeutic activity, comprising probiotic preparations, but also bacteria that get into the upper gastrointestinal tract with water, regular meals and 
partially with air capable of translocation to blood and parenchymal tissue of animals and humans.

Until recently, in studies of comprehensive effect of normal microflora on animals, only microorganisms isolated from the different parts of the digestive tract were studied in detail. At this, the stimulating effect on nonspecific resistance of the macroorganism caused by microbial communities, from the upper gastrointestinal tract that due to the bacterial translocation phenomenon, enters the bloodstream, and correspondingly, organs and tissues was underestimated [17-19].

As it was noted above the phenomenon of bacterial translocation was studied in the D. K. Zabolotny Institute of Microbiology and Virology NAS of Ukraine and described in publications of V. V. Smirnov et al. By that time basic, regularities of this phenomenon were formulated. Other researchers have latterly confirmed and refined these results. Some of the discovered "axioms" of bacterial translocation phenomenon can be stated here: bacteria penetrate into the animals blood within a few minutes after introduction per os; bacterial load (dose) depends not only on bacteria concentration in the blood and parenchymal organs, but also on the duration of their stay in internal environment of the body; about 1/1000 of the total number of bacterial cells entering the digestive tract can be translocated; penetration of bacteria into the blood and animal tissues does not cause any pathological processes that can be detected with modern clinical, laboratory, morphological and other diagnostic methods [20,21].

Importance of bacterial translocation. There is no doubt the importance of studying the translocation phenomenon of probiotic strains of different taxonomic groups (bacilli, colibacilli, bifidus bacteria, lactobacilli, etc.) that form the bases for the creation of veterinary and medical preparations of different kinds of operations (preventive, curative and stimulating), its dynamics, determination of certain reaction of macroorganism by terms of morphological changes in organs and activity of its main systems. This will significantly expand the understanding of the complex process of interaction of probiotics with macroorganism.

Representatives of normal microflora continually upcoming to the microorganism with food and water can have a stronger and versatile influence on animal's body functioning. This includes greater stimulation of nonspecific resistance than it was previously thought, mostly due to the phenomenon of bacterial translocation as an important step towards maintenance of the appropriate level of natural macroorganism defenses.

However, bacteria of upper gastrointestinal tract (oropharynx and stomach) are not the only triggers of nonspecific resistance stimulation of macroorganism at translocation. These processes continue through the complete microorganisms' passage in all parts of the gastrointestinal tract. In addition, biologically active compounds secreted in blood and lymph by the intestine cells in response to bacterial interaction and produced directly by microorganisms [22] cause a great impact on immune system stimulating.

As for the practical importance of expanding and deepening of our understanding of the microorganisms translocation, especially when talking about the probiotic bacteria, it should be noted that nowadays the development of new approaches in strategy and tactics of creation and application of biological preparations of bacterial origin is very urgent. Thus, due to the phenomenon of bacterial translocation, it is possible to develop probiotic 
veterinary and medical preparations intended for the prevention and treatment of not only the disorders of digestive system, but also many diseases of farm animals and humans that are located outside of the gastrointestinal tract [23]. At this, the probiotic bacteria enter the body orally, which simplifies the technology of their application in animal husbandry [8, $24,25]$.

Hence, the analysis of scientific literature shows the lack of available information on bacterial translocation of probiotic bacteria (especially lactobacilli) in domestic and international sources, and therefore, provides grounds for the importance of this phenomenon study in order to increase and accumulate the existing knowledge on the issue. 